\title{
PAIR: Planning and Iterative Refinement in Pre-trained Transformers for Long Text Generation
}

\author{
Xinyu Hua \\ Khoury College of Computer Sciences \\ Northeastern University \\ Boston, MA \\ hua.x@northeastern.edu
}

\author{
Lu Wang \\ Computer Science and Engineering \\ University of Michigan \\ Ann Arbor, MI \\ wangluxy@umich. edu
}

\begin{abstract}
Pre-trained Transformers have enabled impressive breakthroughs in generating long and fluent text, yet their outputs are often "rambling" without coherently arranged content. In this work, we present a novel content-controlled text generation framework, PAIR, with planning and iterative refinement, which is built upon a large model, BART. We first adapt the BERT model to automatically construct the content plans, consisting of keyphrase assignments and their corresponding sentence-level positions. The BART model is employed for generation without modifying its structure. We then propose a refinement algorithm to gradually enhance the generation quality within the sequence-tosequence framework. Evaluation with automatic metrics shows that adding planning consistently improves the generation quality on three distinct domains, with an average of 20 BLEU points and 12 METEOR points improvements. In addition, human judges rate our system outputs to be more relevant and coherent than comparisons without planning.
\end{abstract}

\section{Introduction}

Large pre-trained language models are the cornerstone of many state-of-the-art models in various natural language understanding and generation tasks (Devlin et al., 2019; Liu et al., 2019; Lewis et al., 2020), yet they are far from perfect. In generation tasks, although models like GPT-2 (Radford et al., 2019) are able to produce plausible text, their spontaneous nature limits their utility in actual applications, e.g., users cannot specify what contents to include, and in what order.

To make large models more useful in practice, and to improve their generation quality, we believe it is critical to inform them of when to say what, which is addressed as content planning in traditional generation systems (Duboue and McKeown,

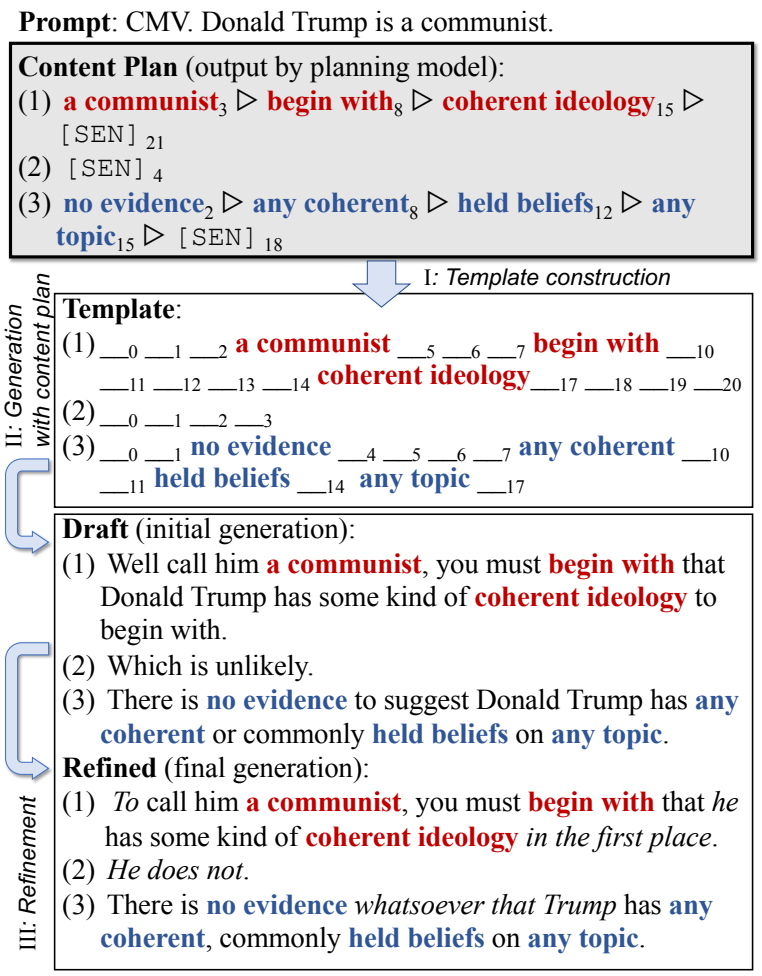

Figure 1: An argument generation example using Reddit ChangeMyView. [Top] Partial output by our planner with keyphrase assignment and positions (in subscripts) for each sentence, segmented by special token [SEN], from which a template is constructed. [Bottom] A draft is first produced and then refined, with updated words highlighted in italics.

2001; Stent et al., 2004). Specially designed control codes and auxiliary planning modules have been integrated into neural models (Keskar et al., 2019; Moryossef et al., 2019; Hua and Wang, 2019), yet those solutions require model architecture modification or retraining, making text generation with large models a very costly endeavor.

To this end, this work aims to bring new insights into how to effectively incorporate content plans into large models to generate more rele- 
vant and coherent text. We first study a planning model trained from BERT (Devlin et al., 2019) to produce the initial content plan, which assigns keyphrases to different sentences and predicts their positions. Next, we propose a contentcontrolled text generation framework, built upon the pre-trained sequence-to-sequence (seq2seq) Transformer model BART (Lewis et al., 2020). As shown in Figure 1, our generation model takes in a content plan consisting of keyphrase assignments and their corresponding positions for each sentence. The plan is encoded as a template, with [MASK] tokens added at positions where no content is specified. Our model then outputs a fluent and coherent multi-sentence text (draft) to reflect the plan. This is done by fine-tuning BART without modifying its architecture.

Furthermore, we present an iterative refinement algorithm to improve the generation in multiple passes, within the seq2seq framework. At each iteration, tokens with low generation confidence are replaced with [MASK] to compose a new template, from which a new output is produced. Unlike prior refinement algorithms that only permit editing in place, our solution offers more flexibility. Figure 1 exemplifies the refinement outcome.

We call our system PAIR (Planning And Iterative Refinement). ${ }^{1}$ It is experimented on three distinct domains: counter-argument generation with Reddit ChangeMyView data, opinion article writing with the New York Times (NYT) corpus $^{2}$ (Sandhaus, 2008), and news report production on NYT. Automatic evaluation with BLEU, ROUGE, and METEOR shows that, by informing the generation model with sentence-level content plans, our model significantly outperforms a BART model fine-tuned with the same set of keyphrases as input ( $§ 5.1)$. Human judges also rate our system outputs as more relevant and coherent ( $(5.2)$. Additionally, our iterative refinement strategy consistently improves the generation quality according to both automatic scores and human evaluation. Finally, our model achieves better content control by reflecting the specified keyphrases in the content plan, whose outputs are preferred by human to another version with weaker control.

To summarize, our major contributions include:

- We propose a novel content planner built upon

\footnotetext{
${ }^{1}$ Code and data are available at: http://xinyuhua . github.io/Resources/emnlp20/

${ }^{2}$ https: //catalog.ldc.upenn.edu/ LDC2008T19
}

BERT to facilitate long-form text generation.

- We present a novel template mask-and-fill method to incorporate content planning into generation models based on BART.

- We devise an iterative refinement algorithm that works within the seq2seq framework to flexibly improve the generation quality.

\section{Related Work}

Content Planning as a Generation Component.

Despite the impressive progress made in many generation tasks, neural systems are known to produce low-quality content (Wiseman et al., 2017; Rohrbach et al., 2018), often with low relevance (Li et al., 2016) and poor discourse structure (Zhao et al., 2017; Xu et al., 2020). Consequently, planning modules are designed and added into neural systems to enhance content relevance (Wiseman et al., 2018; Moryossef et al., 2019; Yao et al., 2019; Hua and Wang, 2019). However, it is still an open question to include content plans in large models, given the additional and expensive model retraining required. This work innovates by adding content plans as masked templates and designing refinement strategy to further boost generation performance, without architectural change.

Controlled Text Generation. Our work is also in line with the study of controllability of neural text generation models. This includes manipulating the syntax (Dušek and Jurčíček, 2016; Goyal and Durrett, 2020) and semantics (Wen et al., 2015; Chen et al., 2019) of the output. Specific applications encourage the model to cover a given topic (Wang et al., 2017; See et al., 2019), mention specified entities (Fan et al., 2018), or display a certain attribute (Hu et al., 2017; Luo et al., 2019; Balakrishnan et al., 2019). However, most existing work relies on model engineering, limiting the generalizability to new domains and adaptability to large pre-trained Transformers. One exception is the Plug and Play model (Dathathri et al., 2020), which directly modifies the key and value states of GPT2 (Radford et al., 2019). However, since the signal is derived from the whole generated text, it is too coarse to provide precise sentence-level content control. Here, we instead gain fine-grained controllability through keyphrase assignment and positioning per sentence, which can be adapted to any off-the-shelf pre-trained Transformer generators.

Iterative Refinement has been studied in machine translation (Lee et al., 2018; Freitag et al., 2019; 
Mansimov et al., 2019; Kasai et al., 2020) to gradually improve translation quality. Refinement is also used with masked language models to improve fluency of non-autoregressive generation outputs (Ghazvininejad et al., 2019; Lawrence et al., 2019). Our work uses BART (Lewis et al., 2020), a state-of-the-art seq2seq model that offers better generalizability and stronger capacity for long text generation. Our proposed strategy substantially differs from prior solutions that rely on in-place word substitutions (Novak et al., 2016; Xia et al., 2017; Weston et al., 2018), as we leverage the seq 2 seq architecture to offer more flexible edits.

\section{Content-controlled Text Generation with PAIR}

Task Description. Our input consists of (1) a sentence-level prompt $\boldsymbol{x}$, such as a news headline, or a proposition in an argument, and (2) a set of keyphrases $\boldsymbol{m}$ that are relevant to the prompt. The system aims to generate $\boldsymbol{y}$ that contains multiple sentences, as in a news report or an argument, by reflecting the keyphrases in a coherent way.

In this section, we first introduce content planning built upon BERT, that assigns keyphrases into sentences and predicts their positions ( $§ 3.1)$. Then we propose a seq2seq generation framework with BART fine-tuning that includes a given content plan derived from keyphrases $\boldsymbol{m}$ (§3.2). Finally, $\S 3.3$ discusses improving generation quality by iteratively masking the less confident predictions and regenerating within our framework.

\subsection{Content Planning with BERT}

Our content planner is trained from BERT to assign keyphrases to different sentences and predict their corresponding positions. As shown in Figure 2 , the concatenation of prompt $\boldsymbol{x}$ and unordered keyphrases $\boldsymbol{m}$ is encoded with bidirectional selfattentions. Keyphrase assignments are produced autoregressively as a sequence of tokens $\boldsymbol{m}^{\prime}=\left\{w_{j}\right\}$, with their positions in the sentence $s=\left\{s_{j}\right\}$ predicted as a sequence tagging task.

We choose BERT because it has been shown to be effective at both language modeling and sequence tagging. Moreover, we leverage its segment embedding to distinguish the input and output sequences. Specifically, we reuse its pre-trained language model output layer for keyphrase assignment. We further design a separate keyphrase positioning layer to predict token position $s_{j}$ as the relative

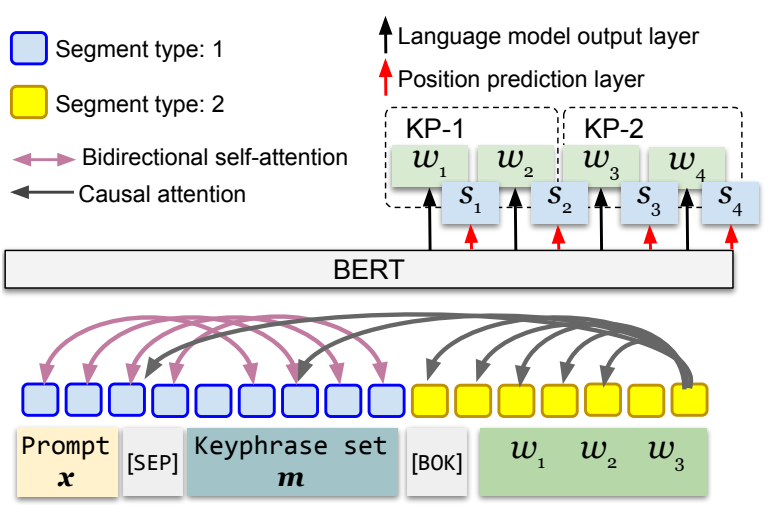

Figure 2: Content planning with BERT. We use bidirectional self-attentions for input encoding, and apply causal self-attentions for keyphrase assignment and position prediction. The input $(\boldsymbol{x}, \boldsymbol{m})$ and output keyphrase assignments $\left(\boldsymbol{m}^{\prime}\right)$ are distinguished by different segment embeddings.

distance from each sentence's beginning:

$$
p\left(s_{j} \mid \boldsymbol{w}_{\leq j}\right)=\operatorname{softmax}\left(\boldsymbol{H}^{L} \boldsymbol{W}_{s}\right)
$$

where $\boldsymbol{H}^{L}$ is the last layer hidden states of the Transformer, and $\boldsymbol{W}_{s}$ are the newly added keyphrase positioning parameters learned during BERT fine-tuning. The range of allowed positions is from 0 to 127 .

Noticeably, as our prediction is done autoregressively, attentions should only consider the generated tokens, but not the future tokens. However, BERT relies on bidirectional self-attentions to attend to both left and right. To resolve this discrepancy, we apply causal attention masks (Dong et al., 2019) over $\boldsymbol{m}^{\prime}$ to disallow attending to the future (gray arrows in Figure 2).

Training the Planner. We extract keyphrases and acquire their ground-truth positions from humanwritten references, and fine-tune BERT with crossentropy losses for both assignment and positioning, with a scaling factor 0.1 over the positioning loss.

Inference. A [BOK] token signals the beginning of keyphrase assignment generation. We employ a greedy decoding algorithm, and limit the output vocabulary to tokens in $\boldsymbol{m}$ and ensure each keyphrase is generated at most once. To allow sentence-level content planning, a special [SEN] token is generated to represent the sentence boundary, with its predicted position indicating the length. The planning process terminates when $[E O S]$ is produced. 


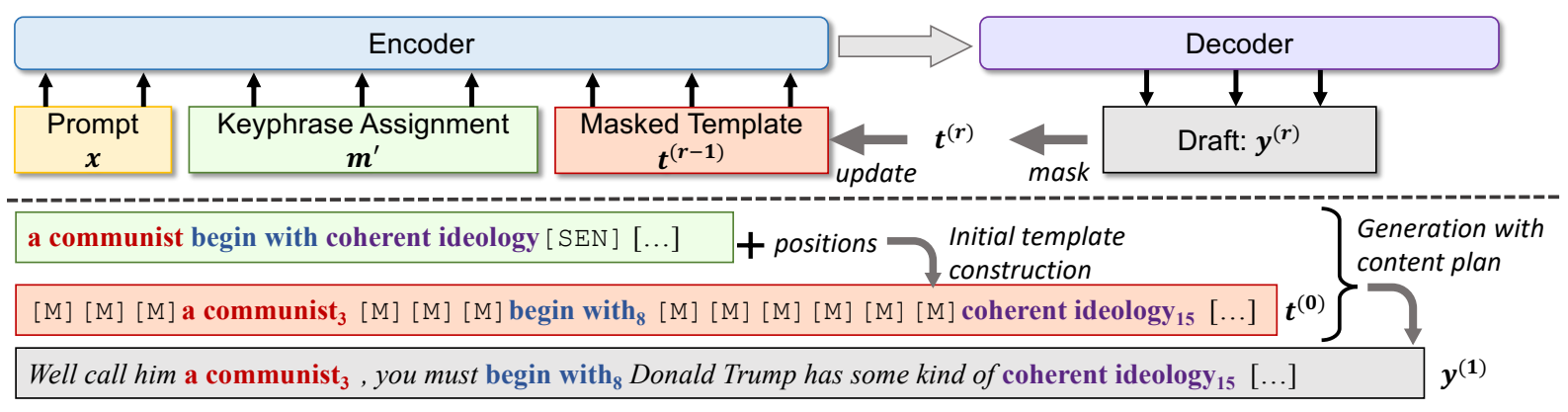

Figure 3: Our content-controlled text generation framework, PAIR, which is built on BART. Decoding is executed iteratively. At each iteration, the encoder consumes the input prompt $\boldsymbol{x}$, the keyphrase assignments $\boldsymbol{m}^{\prime}$, as well as a partially masked template $\left(\boldsymbol{t}^{(r-1)}\right.$ for the $r$-th iteration, [M] for masks). The autoregressive decoder produces a complete sequence $\boldsymbol{y}^{(r)}$, a subset of which is further masked, to serve as the next iteration's template $\boldsymbol{t}^{(r)}$.

\subsection{Adding Content Plan with a Template Mask-and-Fill Procedure}

Given a content planning model, we invoke it to output keyphrase assignments to different sentences $\left(\boldsymbol{m}^{\prime}\right)$, their corresponding positions $s$, along with each sentence's length (based on the prediction of $[S E N]$ ). We first employ a post-processing step to convert between different tokenizers, and correct erroneous position predictions that violate the assignment ordering or break the consecutivity of the phrase (Appendix A). We then convert the plan into a template $\boldsymbol{t}^{(0)}$ as follows: For each sentence, the assigned keyphrases are placed at their predicted positions, and empty slots are filled with [MASK] symbols. Figure 3 illustrates the template construction process and our seq2seq generation model. In Appendix B, we show statistics on the constructed templates.

The input prompt $\boldsymbol{x}$, keyphrase assignments $\boldsymbol{m}^{\prime}$, and template $\boldsymbol{t}^{(0)}$ are concatenated as the input to the encoder. The decoder then generates an output $\boldsymbol{y}^{(1)}$ according to the model's estimation of $p\left(\boldsymbol{y}^{(1)} \mid \boldsymbol{x}, \boldsymbol{m}^{\prime}, \boldsymbol{t}^{(0)}\right) \cdot \boldsymbol{y}^{(1)}$ is treated as a draft, to be further refined as described in the next section.

Our method is substantially different from prior work that uses constrained decoding to enforce words to appear at specific positions (Hokamp and Liu, 2017; Post and Vilar, 2018; Hu et al., 2019), which is highly biased by the surrounding few words and suffers from disfluency. Since BART is trained to denoise the masked input with contextual understanding, it naturally benefits our method.

Decoding. We employ the nucleus sampling strategy (Holtzman et al., 2019), which is shown to yield superior output quality in long text generation. In addition to the standard top- $k$ sampling from tokens with the highest probabilities, nucleus sam- pling further limits possible choices based on a cumulative probability threshold (set to 0.9 in all experiments below). We also require the keyphrases to be generated at or nearby their predicted positions. Concretely, for positions that match any keyphrase token, we force the decoder to copy the keyphrase unless it has already been generated in the previous five tokens. We sample three times to choose the one with the lowest perplexity, as estimated by GPT- $2_{\text {base }}$ (Radford et al., 2019).

\subsection{Iterative Refinement}

Outputs generated in a single pass may suffer from incorrectness and incoherence (see Figure 1), therefore we propose an iterative refinement procedure to improve the quality. In each pass, tokens with low generation confidence are masked (Algorithm 1). This is inspired by iterative decoding designed for inference acceleration in non-autoregressive generation (Lee et al., 2018; Lawrence et al., 2019), though their refinement mostly focuses on word substitution and lacks the flexibility for other operations. Moreover, our goal is to improve fluency while ensuring the generation of given keyphrases.

At each iteration, the $n$ least confident tokens are replaced with [MASK]. Similar as the maskpredict algorithm (Ghazvininejad et al., 2019), we gradually reduce the number of masks. In our experiments, each sample is refined for 5 iterations, with $n$ decaying linearly from $80 \%$ of $\left|\boldsymbol{y}^{(r)}\right|$ to 0 .

Training the Generator. Our training scheme is similar to masked language model pre-training. Given the training corpus $\mathcal{D}=\left\{\left(\boldsymbol{x}_{i}, \boldsymbol{m}_{i}^{\prime}, \boldsymbol{y}_{i}\right)\right\}$, we consider two approaches that add noise to the target $\boldsymbol{y}_{i}$ by randomly masking a subset of (1) any tokens, or (2) tokens that are not within the span 


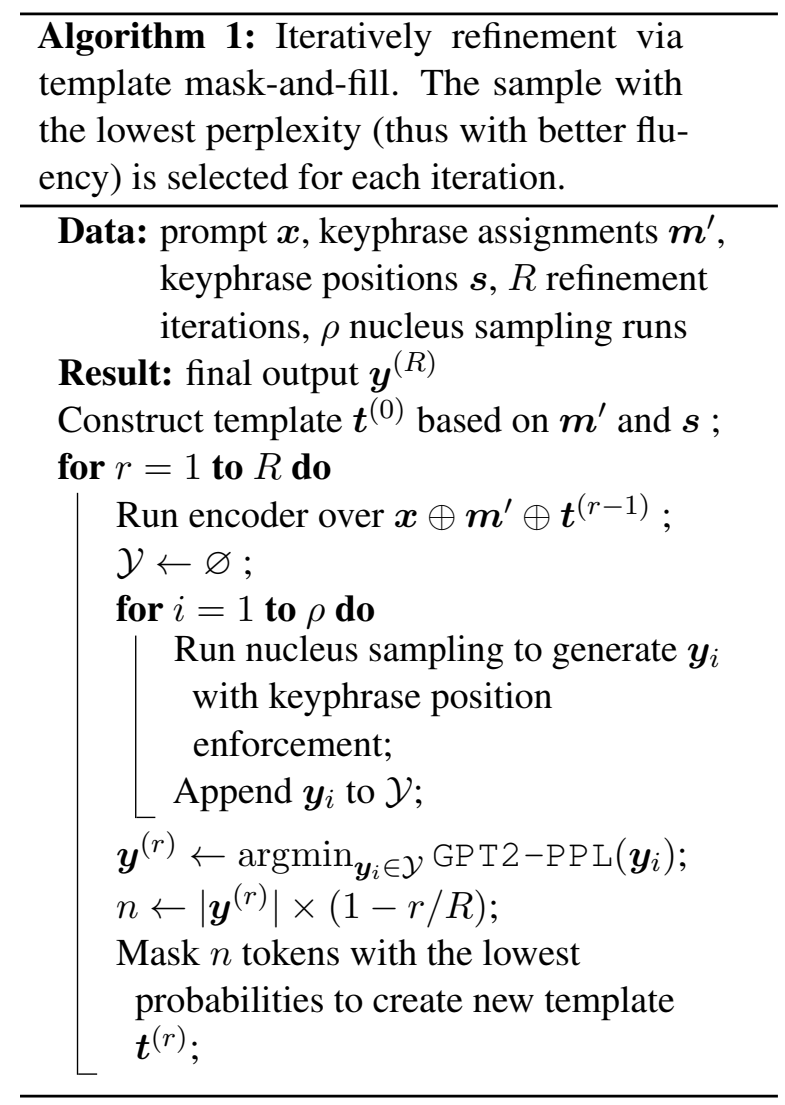

of any keyphrase. The latter is better aligned with our decoding objective, since keyphrases are never masked. We concatenate $\boldsymbol{x}_{i}, \boldsymbol{m}_{i}^{\prime}$, and the corrupted target $\widetilde{\boldsymbol{y}}_{i}$ as input, and fine-tine BART to reconstruct the original $\boldsymbol{y}_{i}$ with a cross-entropy loss.

\section{Experiment Setups}

\subsection{Tasks and Datasets}

We evaluate our generation and planning models on datasets from three distinct domains for multiparagraph-level text generation: (1) argument generation (A RGGEN) (Hua et al., 2019), to produce a counter-argument to refute a given proposition; (2) writing opinionated articles (OPINION), e.g., editorials and op-eds, to show idea exchange on a given subject; and (3) composing news reports (NEWS) to describe events. The three domains are selected with diverse levels of subjectivity and various communicative goals (persuading vs. informing), with statistics shown in Table 1.

Task 1: Argument Generation. We first evaluate our models on persuasive argument generation, based on a dataset collected from Reddit $r$ /ChangeMyView (CMV) in our prior work (Hua et al., 2019). This dataset contains pairs of original post (OP) statement on a contro-

\begin{tabular}{llllll}
\hline \multicolumn{7}{c}{ \# Sample } & $\mid$ Prompt & $\mid$ Target & \# KP KP Cov. \\
\hline ARGGEN 56,504 & 19.4 & 116.6 & 20.6 & $30.5 \%$ \\
OPINION & 104,610 & 6.1 & 205.6 & 19.0 & $26.0 \%$ \\
NEWS & 239,959 & 7.0 & 282.7 & 30.3 & $32.6 \%$ \\
\hline
\end{tabular}

Table 1: Statistics of the three datasets. We report average lengths of the prompt and the target generation, number of unique keyphrases (\# KP) used in the input, and the percentage of content words in target covered by the keyphrases (KP Cov.).

versial issue about politics and filtered high-quality counter-arguments, covering 14, 833 threads from 2013 to 2018 . We use the OP title, which contains a proposition (e.g. the minimum wage should be abolished), to form the input prompt $\boldsymbol{x}$. In our prior work, only the first paragraphs of high-quality counter-arguments are used for generation. Here we consider generating the full post, which is significantly longer. Keyphrases are identified as noun phrases and verb phrases that contain at least one topic signature word (Lin and Hovy, 2000), which is determined by a log-likelihood ratio test that indicates word salience. Following our prior work, we expand the set of topic signatures with their synonyms, hyponyms, hypernyms, and antonyms according to WordNet (Miller, 1994). The keyphrases longer than 10 tokens are further discarded.

Task 2: Opinion Article Generation. We collect opinion articles from the New York Times (NYT) corpus (Sandhaus, 2008). An article is selected if its taxonomies label has a prefix of Top/Opinion. We eliminate articles with an empty headline or less than three sentences. Keyphrases are extracted in a similar manner as done in argument generation. Samples without any keyphrase are removed. The article headline is treated as the input, and our target is to construct the full article. Table 1 shows that opinion samples have shorter input than arguments, and the keyphrase set also covers fewer content words in the target outputs, requiring the model to generalize well to capture the unseen tokens.

Task 3: News Report Generation. Similarly, we collect and process news reports from NYT, filtering by taxonomy labels starting with "Top/News", removing articles that have no content word overlap with the headline, and ones with material-types labeled as one of "statistics", "list", "correction", "biography", or "review." News reports describe events and facts, and in this domain we aim to study and emphasize the impor- 


\begin{tabular}{|c|c|c|c|c|c|c|c|c|c|c|c|c|}
\hline & \multicolumn{4}{|c|}{ ARGGEN } & \multicolumn{4}{|c|}{ OPINION } & \multicolumn{4}{|c|}{ NEWS } \\
\hline & B-4 & R-L & MTR & Len. & B-4 & R-L & MTR & Len. & B-4 & R-L & MTR & Len. \\
\hline $\mathrm{SEQ} 2$ & 0.76 & 13.80 & 9.36 & 97 & 1.42 & 15.97 & 10.97 & 156 & 1.11 & 15.60 & 10.10 & 242 \\
\hline KPSEQ2SEQ & 6.78 & 19.43 & 15.98 & 97 & 11.38 & 22.75 & 18.38 & 164 & 11.61 & 21.05 & 18.61 & 286 \\
\hline$\overline{\mathrm{PAI}}_{\text {light }}^{-}-$ & $-\overline{6} . \overline{3} 8$ & $\overline{4} \overline{7.97}$ & $\overline{31} . \overline{6}$ & ${ }^{-} 1 \overline{1} 9^{-}$ & $1 \overline{6} . \overline{2} \overline{7}$ & $\overline{3} \overline{3.30}$ & $\overline{24.3 \overline{2}}$ & $2 \overline{1} 0$ & $2 \overline{8} . \overline{0} 3$ & $\overline{4} \overline{3.39}$ & $27 . \overline{7}$ & $2 \overline{7} \overline{2}$ \\
\hline PAIR $_{\text {light }}$ w/o refine & 25.17 & 46.84 & 31.31 & 120 & 15.45 & 32.35 & 24.11 & 214 & 27.32 & 43.08 & 27.35 & 278 \\
\hline PAIR full $_{\text {fill }}$ & 36.09 & 56.86 & 33.30 & 102 & 23.12 & 40.53 & 24.73 & 167 & 34.37 & 51.10 & 29.50 & 259 \\
\hline PAIR full $_{\text {w/o refine }}$ & 34.09 & 55.42 & 32.74 & 101 & 22.17 & 39.71 & 24.65 & 169 & 33.48 & 50.27 & 29.26 & 260 \\
\hline
\end{tabular}

Table 2: Key results on argument generation, opinion article writing, and news report generation. BLEU-4 (B4), ROUGE-L (R-L), METEOR (MTR), and average output lengths are reported (for references, the lengths are

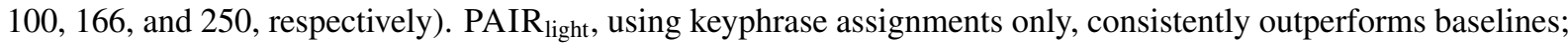
adding keyphrase positions, PAIR full further boosts scores. Improvements by our models over baselines are all significant ( $p<0.0001$, approximate randomization test). Iterative refinement helps on both setups.

tance of faithfully reflecting content plans during generation and refinement.

Data Split and Preprocessing. For argument generation, we split the data into $75 \%, 12.5 \%$, and $12.5 \%$ for training, validation, and test sets. To avoid test set contamination, the split is conducted on thread level. For opinion and news generation, we reserve the most recent $5 \mathrm{k}$ articles for testing, another $5 \mathrm{k}$ for validation, and the rest (23k for news and 10k for opinion) are used for training. We apply the BPE tokenization (Sennrich et al., 2016) for the generation model as BART does, and use WordPiece (Wu et al., 2016) for BERT-based planner. To fit the data into our GPUs, we truncate the target size to 140 tokens for argument, sizes of 243 and 335 are applied for opinion and news, for both training and inference.

\subsection{Implementation Details}

Our code is written in PyTorch (Paszke et al., 2019). For fine-tuning, we adopt the standard linear warmup and inverse square root decaying scheme for learning rates, with a maximum value of $5 \times 10^{-5}$. Adam (Kingma and Ba, 2014) is used as the optimizer, with a batch size of 10 for refinement and 20 for content planning, and a maximum gradient clipped at 1.0. All hyperparameters are tuned on validation set, with early stopping used to avoid overfitting. More details are in Appendix A.

\subsection{Baselines and Comparisons}

We consider two baselines, both are fine-tuned from BART as in our models: (1) SEQ2SEQ directly generates the target from the prompt; (2) KPSEQ2SEQ encodes the concatenation of the prompt and the unordered keyphrase set. To study if using only sentence-level keyphrase assignments helps, we include a model variant (PAIR $\left.\mathbf{P}_{\text {light }}\right)$ by removing keyphrase position information $(s)$ from the input of our generator and using an initial template with all [MASK] symbols. Our model with full plans is denoted as PAIR full. We first report generation results using ground-truth content plans constructed from human-written text, and also show the end-to-end results with predicted content plans by our planner.

\section{Results}

\subsection{Automatic Evaluation}

We report scores with BLEU (Papineni et al., 2002), which is based on n-gram precision (up to 4-grams); ROUGE-L (Lin, 2004), measuring recall of the longest common subsequences; and METEOR (Lavie and Agarwal, 2007), which accounts for paraphrase. For our models PAIR full $_{\text {and }}$ PAIR light, we evaluate both the first draft and the final output after refinement. Table 2 lists the results when ground-truth content plans are applied.

First, our content-controlled generation model with planning consistently outperforms comparisons and other model variants on all datasets, with or without iterative refinement. Among our

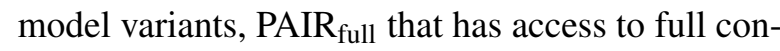
tent plans obtains significantly better scores than PAIR ${ }_{\text {light }}$ that only includes keyphrase assignments but not their positions. Lengths of PAIR full's outputs are also closer to those of human references. Both imply the benefit of keyphrase positioning.

Table 2 also shows that the iterative refinement strategy can steadily boost performance on both of our setups. By inspecting the performance of refinement in different iterations (Figure 4), we observe that both BLEU and ROUGE-L scores gradually increase while perplexity lowers as the 


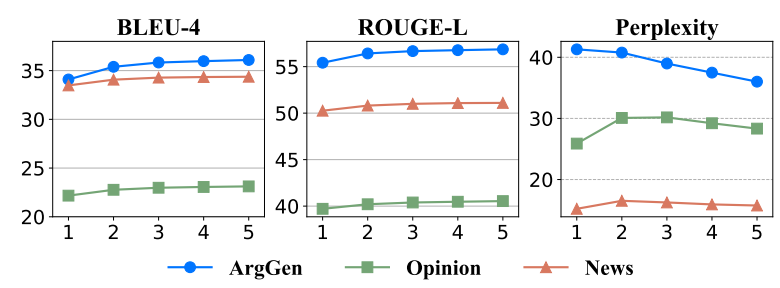

Figure 4: Results on iterative refinement with five iterations. Both BLEU and ROUGE-L scores steadily increase, with perplexity lowers in later iterations.

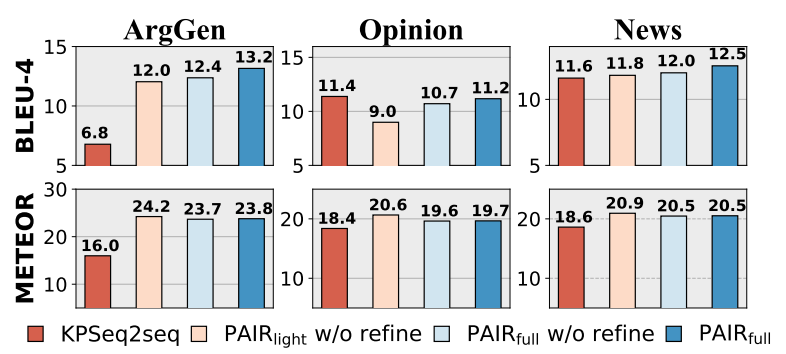

Figure 5: End-to-end generation results with automatically predicted content plans. Our models outperform KPSEQ2SEQ in both metrics, except for BLEU-4 on opinion articles where results are comparable.

refinement progresses. This indicates that iterative post-editing improves both content and fluency.

Results with Predicted Content Plans. We further report results by using content plans predicted by our BERT-based planner. Figure 5 compares PAIR $_{\text {full }}$ and PAIR light with KPSEQ2SEQ. Our models yield better METEOR scores on all three domains. That said, the improvement from predicted plans is not as pronounced as that from ground-truth plans. Upon inspection, we find that our planner often falls short of accurately positioning the given keyphrases, leading to degraded generation performance. This points to a potential direction for future work where better positioning model should be developed.

\subsection{Human Evaluation}

We hire four proficient English speakers ${ }^{3}$ to rate three aspects of the generated arguments on a scale of 1 (worst) to 5 (best): fluency, coherence-if the information organization is natural and logical, and relevance - if the topic is related to the prompt and whether the stance is correct. 50 samples are randomly selected, with system outputs by KPSEQ2SEQ, PAIR full and PAIR ${ }_{\text {light }}$ shown to human judges in random order. The evaluation

\footnotetext{
${ }^{3}$ They are all US-based college students. Each of them is paid $\$ 15$ hourly for the task.
}

\begin{tabular}{|c|c|c|c|}
\hline ARGGEN & Fluency & Coherence & Relevance \\
\hline KPSEQ2SEQ & 4.63 & 3.28 & 2.79 \\
\hline$-\overline{\mathrm{PA}} \overline{\mathrm{I}} \overline{\mathrm{R}}_{\text {light }}-\overline{-}$ & $\overline{4.75}$ & $3.97^{*}$ & $\overline{3.85}^{*}$ \\
\hline PAIR $_{\text {full }}$ & 4.46 & $3.76^{*}$ & $3.79^{*}$ \\
\hline
\end{tabular}

Table 3: Human evaluation for argument generation on fluency, coherence, and relevance, with 5 as the best. The Krippendorff's $\alpha$ are $0.28,0.30$, and 0.37 , respectively. Our model outputs are significantly more coherent and relevant than KPSEQ2SEQ $\left(^{*}: p<0.0001\right)$, with comparable fluency.

Prompt (News): 4 Arrested in Theft of Baby Jesus Figurines

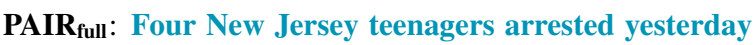
were accused of stealing more than 25 plastic baby Jesus figurines from a church before they burn in a bonfire, the police said. The police in Sayreville, N.J., arrested Michael Payne, 18, and T.J. Jones, 18, of Union City; Nicholas M. Hess, 18, of Matawan; and Jason L. O'Neill, 18, of Port New York, N.J., and charged them with burglary. Their vandals removed more than 100 figurines of Jesus from a cemetery outside St. Stanislaus Kostka Church in Sayreville, the police said. Detectives said the four had taken their flashlights to the cemetery and jotted down the license plates of several cars in the vicinity [...]

Prompt (Opinion): Drowning in a Sea of College Debt

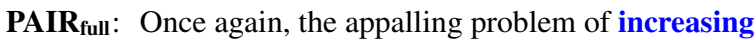
debt in financing higher education that has taken place is more intolerable, particularly because of the arrogance of our colleges and universities. The burden of saddling college students with debt in the middle of their teenage years, when they were in debt, is essential for a good education. Our educational system is designed to allow kids to develop the skills necessary, but it does not create optimal conditions for mature students who know they will not be able [...]

Table 4: Sample outputs in the news and opinion domain. Keyphrases assigned to different sentences are in boldface and color-coded.

guideline is in the supplementary material.

Table 3 shows that both of our models are rated with better coherence and relevance than KPSEQ2SEQ which uses the same but unordered keyphrases as input. Interestingly, outputs by PAIR $_{\text {light }}$ are regarded as more fluent and coherent, though the difference is not significant. However, discourse analysis in $\S 6$ reveals that clauses produced by PAIR light $_{\text {are more locally related, com- }}$ pared to PAIR full, which can be perceived as easier to read. In addition to the sample argument in Figure 1, Table 4 shows PAIR full's output in the news and opinion domains. More samples by different systems are in the supplementary material.

Effect of Refinement and Keyphrase Enforce- 
ment. We further ask whether human judges prefer the refined text and whether enforcing keyphrases to be generated yields noticeable content improvement. In a second study, we present the same 50 prompts from the previous evaluation on argument generation, and an additional 50 samples for opinion article writing to the same group of human judge. For each sample, PAIR full's outputs with and without refinement are shown in random order. Judges indicate their preference based on the overall quality. The same procedure is conducted to compare with a version where we do not enforce keyphrases to be copied at their predicted positions during decoding. Table 5 demonstrates that the refined text is preferred in more than half of the cases, for both domains. Enforcing keyphrase generation based on their positions is also more favorable than not enforcing such constraint.

\begin{tabular}{lcc|cc} 
& PAIR $_{\text {full }}$ & w/o refine & PAIR $_{\text {full }}$ w/o enforce \\
ARGGEN & $\mathbf{5 2 . 7 \%}$ & $33.3 \%$ & $\mathbf{4 5 . 3 \%}$ & $40.0 \%$ \\
OPINION & $\mathbf{5 2 . 7 \%}$ & $30.7 \%$ & $\mathbf{5 0 . 0 \%}$ & $29.3 \%$
\end{tabular}

Table 5: Percentages of samples preferred by human judges before and after refinement [Left]; with and without enforcing keyphrases to appear at the predicted positions [Right]. Ties are omitted.

What is updated during iterative refinement? Since refinement yields better text, we compare generations before and after the refinement. First, we find that masks are regularly put on "functional" words and phrases. For example, stopwords and punctuation along with their bigrams are often swapped out, with new words filled in to improve fluency. Moreover, about $85 \%$ of the refinement operations result in new content being generated. This includes changing prepositions and paraphrasing, e.g., replacing "a research fellow" with "a graduate student." On both news and opinion domains, numerical and temporal expressions are often incorrectly substituted, suggesting that better fact control needs to be designed to maintain factuality.

\section{Further Discussions on Discourse}

Prior work's evaluation mainly focuses on fluency and content relevance, and largely ignores the discourse structure exposed by the generated text. However, unnatural discourse and lack of focus are indeed perceived as major problems of longform neural generations, as identified by human ex-

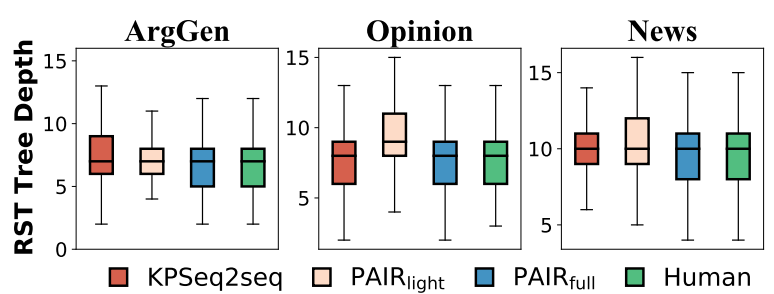

Figure 6: Distributions of RST tree depth. PAIR full better resembles the patterns in human-written texts.

perts. ${ }^{4}$ Here, we aim to investigate whether contentcontrolled generation with ground-truth content plans resembles human-written text by studying discourse phenomena.

Are PAIR generations similar to humanwritten text in discourse structure? We utilize DPLP (Ji and Eisenstein, 2014), an off-theshelf Rhetorical Structure Theory (RST) discourse parser. DPLP converts a given text into a binary tree, with elementary discourse units (EDUs, usually clauses) as nucleus and satellite nodes. For instance, a relation NS-elaboration indicates the second node as a satellite (S) elaborating on the first nucleus (N) node. DPLP achieves F1 scores of 81.6 for EDU detection and 71.0 for relation prediction on news articles from the annotated RST Discourse Treebank (Carlson et al., 2001). We run this trained model on our data for both human references and model generations.

First, we analyze the depth of RST parse trees, which exhibits whether the text is more locally or globally connected. For all trees, we truncate at a maximum number of EDUs based on the 90 percentile of EDU count for human references. Distributions of tree depth are displayed in Figure 6. As can be seen, generations by PAIR full show similar patterns to human-written arguments and articles. We also find that trees by PAIR light $_{\text {tend to have }}$ a more "linear" structure, highlighting the dominance of local relations between adjacent EDUs, compared with PAIR full which uses knowledge of keyphrases positions. This implies that content positioning helps with structure at a more global level. We further look into the ratios of NS, NN, SN relations, and observe that most model outputs have similar trends as human-written texts, except for KPSEQ2SEQ which has more SN relations, e.g., it produces twice as many SNs than others on arguments.

\footnotetext{
${ }^{4}$ https://www.economist.com/open-future/2019/10/01/ how-to-respond-to-climate-change-if-you-are-an-algorithm
} 


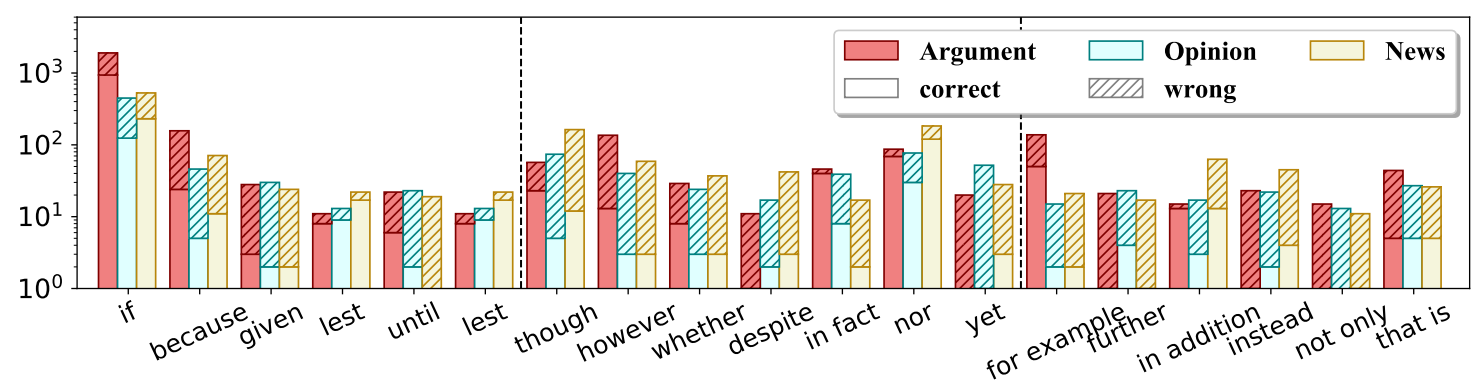

Figure 7: Discourse markers that are correctly and incorrectly (shaded) generated by PAIR full, compared to aligned sentences in human references. Discourse markers are grouped (from left to right) into senses of CONTINGENCY (higher marker generation accuracy observed), COMPARISON, and EXPANSION. $y$-axis: \# of generated sentences with the corresponding marker.

Can PAIR correctly generate discourse markers? Since discourse markers are crucial for coherence (Grote and Stede, 1998; Callaway, 2003) and have received dedicated research efforts in rulebased systems (Reed et al., 2018; Balakrishnan et al., 2019), we examine if PAIR full can properly generate them. For each sample, we construct sentence pairs based on content word overlaps between system generation and human reference. We manually select a set of unambiguous discourse markers from Appendix A of the Penn Discourse Treebank manual (Prasad et al., 2008). When a marker is present in the first three words in a reference sentence, we check if the corresponding system output does the same.

Figure 7 displays the numbers of generated sentences with markers produced as the same in human references (correct) or not (wrong). The markers are grouped into three senses: CONTINGENCY, COMPARISON, and EXPANSION. The charts indicates that PAIR full does better at reproducing markers for CONTINGENCY, followed by COMPARISON and EXPANSION. Manual inspections show that certain missed cases are in fact plausible replacements, such as using at the same time for in addition, or also for further, while in other cases the markers tend to be omitted. Overall, we believe that content control alone is still insufficient to capture discourse relations, motivating future work on discourse planning.

\section{Ethics Statement}

We recognize that the proposed system can generate fabricated and inaccurate information due to the systematic biases introduced during model pretraining based on web corpora. We urge the users to cautiously examine the ethical implications of the generated output in real world applications.

\section{Conclusion}

We present a novel content-controlled generation framework that adds content planning to large pretrained Transformers without modifying model architecture. A BERT-based planning model is first designed to assign and position keyphrases into different sentences. We then investigate an iterative refinement algorithm that works with the sequenceto-sequence models to improve generation quality with flexible editing. Both automatic evaluation and human judgments show that our model with planning and refinement enhances the relevance and coherence of the generated content.

\section{Acknowledgements}

This research is supported in part by National Science Foundation through Grant IIS-1813341 and Nvidia GPU gifts. We thank three anonymous reviewers for their constructive suggestions on many aspects of this work.

\section{References}

Anusha Balakrishnan, Jinfeng Rao, Kartikeya Upasani, Michael White, and Rajen Subba. 2019. Constrained decoding for neural NLG from compositional representations in task-oriented dialogue. In Proceedings of the 57th Annual Meeting of the Association for Computational Linguistics, pages 831844, Florence, Italy. Association for Computational Linguistics.

Charles B. Callaway. 2003. Integrating discourse markers into a pipelined natural language generation architecture. In Proceedings of the 41st Annual Meeting of the Association for Computational Linguistics, pages 264-271, Sapporo, Japan. Association for Computational Linguistics. 
Lynn Carlson, Daniel Marcu, and Mary Ellen Okurovsky. 2001. Building a discourse-tagged corpus in the framework of Rhetorical Structure Theory. In Proceedings of the Second SIGdial Workshop on Discourse and Dialogue.

Mingda Chen, Qingming Tang, Sam Wiseman, and Kevin Gimpel. 2019. A multi-task approach for disentangling syntax and semantics in sentence representations. In Proceedings of the 2019 Conference of the North American Chapter of the Association for Computational Linguistics: Human Language Technologies, Volume 1 (Long and Short Papers), pages 2453-2464, Minneapolis, Minnesota. Association for Computational Linguistics.

Sumanth Dathathri, Andrea Madotto, Janice Lan, Jane Hung, Eric Frank, Piero Molino, Jason Yosinski, and Rosanne Liu. 2020. Plug and play language models: A simple approach to controlled text generation. In International Conference on Learning Representations.

Jacob Devlin, Ming-Wei Chang, Kenton Lee, and Kristina Toutanova. 2019. BERT: Pre-training of deep bidirectional transformers for language understanding. In Proceedings of the 2019 Conference of the North American Chapter of the Association for Computational Linguistics: Human Language Technologies, Volume 1 (Long and Short Papers), pages 4171-4186, Minneapolis, Minnesota. Association for Computational Linguistics.

Li Dong, Nan Yang, Wenhui Wang, Furu Wei, Xiaodong Liu, Yu Wang, Jianfeng Gao, Ming Zhou, and Hsiao-Wuen Hon. 2019. Unified language model pre-training for natural language understanding and generation. In Advances in Neural Information Processing Systems, pages 13042-13054.

Pablo A. Duboue and Kathleen R. McKeown. 2001. Empirically estimating order constraints for content planning in generation. In Proceedings of the 39th Annual Meeting of the Association for Computational Linguistics, pages 172-179, Toulouse, France. Association for Computational Linguistics.

Ondřej Dušek and Filip Jurčíček. 2016. Sequence-tosequence generation for spoken dialogue via deep syntax trees and strings. In Proceedings of the 54th Annual Meeting of the Association for Computational Linguistics (Volume 2: Short Papers), pages 45-51, Berlin, Germany. Association for Computational Linguistics.

WA Falcon. 2019. Pytorch lightning. GitHub. Note: https://github. com/williamFalcon/pytorch-lightning Cited by, 3.

Angela Fan, David Grangier, and Michael Auli. 2018 Controllable abstractive summarization. In Proceedings of the 2nd Workshop on Neural Machine Translation and Generation, pages 45-54, Melbourne, Australia. Association for Computational Linguistics.
Markus Freitag, Isaac Caswell, and Scott Roy. 2019. APE at scale and its implications on MT evaluation biases. In Proceedings of the Fourth Conference on Machine Translation (Volume 1: Research Papers), pages 34-44, Florence, Italy. Association for Computational Linguistics.

Marjan Ghazvininejad, Omer Levy, Yinhan Liu, and Luke Zettlemoyer. 2019. Mask-predict: Parallel decoding of conditional masked language models. In Proceedings of the 2019 Conference on Empirical Methods in Natural Language Processing and the 9th International Joint Conference on Natural Language Processing (EMNLP-IJCNLP), pages 61126121, Hong Kong, China. Association for Computational Linguistics.

Tanya Goyal and Greg Durrett. 2020. Neural syntactic preordering for controlled paraphrase generation. In Proceedings of the 58th Annual Meeting of the Association for Computational Linguistics, pages 238252, Online. Association for Computational Linguistics.

Brigitte Grote and Manfred Stede. 1998. Discourse marker choice in sentence planning. In Natural Language Generation.

Chris Hokamp and Qun Liu. 2017. Lexically constrained decoding for sequence generation using grid beam search. In Proceedings of the 55th Annual Meeting of the Association for Computational Linguistics (Volume 1: Long Papers), pages 1535-1546, Vancouver, Canada. Association for Computational Linguistics.

Ari Holtzman, Jan Buys, Li Du, Maxwell Forbes, and Yejin Choi. 2019. The curious case of neural text degeneration. In International Conference on Learning Representations.

J. Edward Hu, Huda Khayrallah, Ryan Culkin, Patrick Xia, Tongfei Chen, Matt Post, and Benjamin Van Durme. 2019. Improved lexically constrained decoding for translation and monolingual rewriting. In Proceedings of the 2019 Conference of the North American Chapter of the Association for Computational Linguistics: Human Language Technologies, Volume 1 (Long and Short Papers), pages 839-850, Minneapolis, Minnesota. Association for Computational Linguistics.

Zhiting $\mathrm{Hu}$, Zichao Yang, Xiaodan Liang, Ruslan Salakhutdinov, and Eric P Xing. 2017. Toward controlled generation of text. In Proceedings of the 34th International Conference on Machine Learning-Volume 70, pages 1587-1596. JMLR. org.

Xinyu Hua, Zhe Hu, and Lu Wang. 2019. Argument generation with retrieval, planning, and realization. In Proceedings of the 57th Annual Meeting of the Association for Computational Linguistics, pages 2661-2672, Florence, Italy. Association for Computational Linguistics. 
Xinyu Hua and Lu Wang. 2019. Sentence-level content planning and style specification for neural text generation. In Proceedings of the 2019 Conference on Empirical Methods in Natural Language Processing and the 9th International Joint Conference on Natural Language Processing (EMNLP-IJCNLP), pages 591-602, Hong Kong, China. Association for Computational Linguistics.

Yangfeng Ji and Jacob Eisenstein. 2014. Representation learning for text-level discourse parsing. In Proceedings of the 52nd Annual Meeting of the Association for Computational Linguistics (Volume 1: Long Papers), pages 13-24, Baltimore, Maryland. Association for Computational Linguistics.

Jungo Kasai, James Cross, Marjan Ghazvininejad, and Jiatao Gu. 2020. Non-autoregressive machine translation with disentangled context transformer. In Proc. of ICML.

Nitish Shirish Keskar, Bryan McCann, Lav R Varshney, Caiming Xiong, and Richard Socher. 2019. Ctrl: A conditional transformer language model for controllable generation. arXiv preprint arXiv:1909.05858.

Diederik P Kingma and Jimmy Ba. 2014. Adam: A method for stochastic optimization.

Alon Lavie and Abhaya Agarwal. 2007. METEOR: An automatic metric for MT evaluation with high levels of correlation with human judgments. In Proceedings of the Second Workshop on Statistical Machine Translation, pages 228-231, Prague, Czech Republic. Association for Computational Linguistics.

Carolin Lawrence, Bhushan Kotnis, and Mathias Niepert. 2019. Attending to future tokens for bidirectional sequence generation. In Proceedings of the 2019 Conference on Empirical Methods in Natural Language Processing and the 9th International Joint Conference on Natural Language Processing (EMNLP-IJCNLP), pages 1-10, Hong Kong, China. Association for Computational Linguistics.

Jason Lee, Elman Mansimov, and Kyunghyun Cho. 2018. Deterministic non-autoregressive neural sequence modeling by iterative refinement. In Proceedings of the 2018 Conference on Empirical Methods in Natural Language Processing, pages 11731182, Brussels, Belgium. Association for Computational Linguistics.

Mike Lewis, Yinhan Liu, Naman Goyal, Marjan Ghazvininejad, Abdelrahman Mohamed, Omer Levy, Veselin Stoyanov, and Luke Zettlemoyer. 2020. BART: Denoising sequence-to-sequence pretraining for natural language generation, translation, and comprehension. In Proceedings of the 58th Annual Meeting of the Association for Computational Linguistics, pages 7871-7880, Online. Association for Computational Linguistics.

Jiwei Li, Michel Galley, Chris Brockett, Jianfeng Gao, and Bill Dolan. 2016. A diversity-promoting objective function for neural conversation models. In
Proceedings of the 2016 Conference of the North American Chapter of the Association for Computational Linguistics: Human Language Technologies, pages 110-119, San Diego, California. Association for Computational Linguistics.

Chin-Yew Lin. 2004. ROUGE: A package for automatic evaluation of summaries. In Text Summarization Branches Out, pages 74-81, Barcelona, Spain. Association for Computational Linguistics.

Chin-Yew Lin and Eduard Hovy. 2000. The automated acquisition of topic signatures for text summarization. In COLING 2000 Volume 1: The 18th International Conference on Computational Linguistics.

Yinhan Liu, Myle Ott, Naman Goyal, Jingfei Du, Mandar Joshi, Danqi Chen, Omer Levy, Mike Lewis, Luke Zettlemoyer, and Veselin Stoyanov. 2019. Roberta: A robustly optimized bert pretraining approach. arXiv preprint arXiv:1907.11692.

Fuli Luo, Damai Dai, Pengcheng Yang, Tianyu Liu, Baobao Chang, Zhifang Sui, and Xu Sun. 2019. Learning to control the fine-grained sentiment for story ending generation. In Proceedings of the 57th Annual Meeting of the Association for Computational Linguistics, pages 6020-6026, Florence, Italy. Association for Computational Linguistics.

Elman Mansimov, Alex Wang, Sean Welleck, and Kyunghyun Cho. 2019. A generalized framework of sequence generation with application to undirected sequence models. arXiv preprint arXiv:1905.12790.

George A. Miller. 1994. WordNet: A lexical database for English. In Human Language Technology: Proceedings of a Workshop held at Plainsboro, New Jersey, March 8-11, 1994.

Amit Moryossef, Yoav Goldberg, and Ido Dagan. 2019. Step-by-step: Separating planning from realization in neural data-to-text generation. In Proceedings of the 2019 Conference of the North American Chapter of the Association for Computational Linguistics: Human Language Technologies, Volume 1 (Long and Short Papers), pages 2267-2277, Minneapolis, Minnesota. Association for Computational Linguistics.

Roman Novak, Michael Auli, and David Grangier. 2016. Iterative refinement for machine translation. arXiv preprint arXiv:1610.06602.

Kishore Papineni, Salim Roukos, Todd Ward, and WeiJing Zhu. 2002. Bleu: a method for automatic evaluation of machine translation. In Proceedings of the 40th Annual Meeting of the Association for Computational Linguistics, pages 311-318, Philadelphia, Pennsylvania, USA. Association for Computational Linguistics.

Adam Paszke, Sam Gross, Francisco Massa, Adam Lerer, James Bradbury, Gregory Chanan, Trevor Killeen, Zeming Lin, Natalia Gimelshein, Luca Antiga, et al. 2019. Pytorch: An imperative style, 
high-performance deep learning library. In $A d$ vances in Neural Information Processing Systems, pages 8024-8035.

Matt Post and David Vilar. 2018. Fast lexically constrained decoding with dynamic beam allocation for neural machine translation. In Proceedings of the 2018 Conference of the North American Chapter of the Association for Computational Linguistics: $\mathrm{Hu}$ man Language Technologies, Volume 1 (Long Papers), pages 1314-1324, New Orleans, Louisiana. Association for Computational Linguistics.

Rashmi Prasad, Nikhil Dinesh, Alan Lee, Eleni Miltsakaki, Livio Robaldo, Aravind K Joshi, and Bonnie L Webber. 2008. The penn discourse treebank 2.0. In LREC. Citeseer.

Alec Radford, Jeffrey Wu, Rewon Child, David Luan, Dario Amodei, and Ilya Sutskever. 2019. Language models are unsupervised multitask learners. OpenAI Blog, 1(8):9.

Lena Reed, Shereen Oraby, and Marilyn Walker. 2018. Can neural generators for dialogue learn sentence planning and discourse structuring? In Proceedings of the 11th International Conference on Natural Language Generation, pages 284-295, Tilburg University, The Netherlands. Association for Computational Linguistics.

Anna Rohrbach, Lisa Anne Hendricks, Kaylee Burns, Trevor Darrell, and Kate Saenko. 2018. Object hallucination in image captioning. In Proceedings of the 2018 Conference on Empirical Methods in Natural Language Processing, pages 4035-4045, Brussels, Belgium. Association for Computational Linguistics.

Evan Sandhaus. 2008. The new york times annotated corpus. Linguistic Data Consortium, Philadelphia, 6(12):e26752.

Abigail See, Stephen Roller, Douwe Kiela, and Jason Weston. 2019. What makes a good conversation? how controllable attributes affect human judgments. In Proceedings of the 2019 Conference of the North American Chapter of the Association for Computational Linguistics: Human Language Technologies, Volume 1 (Long and Short Papers), pages 1702-1723, Minneapolis, Minnesota. Association for Computational Linguistics.

Rico Sennrich, Barry Haddow, and Alexandra Birch. 2016. Neural machine translation of rare words with subword units. In Proceedings of the 54th Annual Meeting of the Association for Computational Linguistics (Volume 1: Long Papers), pages 17151725, Berlin, Germany. Association for Computational Linguistics.

Amanda Stent, Rashmi Prasad, and Marilyn Walker. 2004. Trainable sentence planning for complex information presentations in spoken dialog systems. In Proceedings of the 42nd Annual Meeting of the Association for Computational Linguistics (ACL04), pages 79-86, Barcelona, Spain.

Di Wang, Nebojsa Jojic, Chris Brockett, and Eric Nyberg. 2017. Steering output style and topic in neural response generation. In Proceedings of the 2017 Conference on Empirical Methods in Natural Language Processing, pages 2140-2150, Copenhagen, Denmark. Association for Computational Linguistics.

Tsung-Hsien Wen, Milica Gašić, Nikola Mrkšić, PeiHao Su, David Vandyke, and Steve Young. 2015. Semantically conditioned LSTM-based natural language generation for spoken dialogue systems. In Proceedings of the 2015 Conference on Empirical Methods in Natural Language Processing, pages 1711-1721, Lisbon, Portugal. Association for Computational Linguistics.

Jason Weston, Emily Dinan, and Alexander Miller. 2018. Retrieve and refine: Improved sequence generation models for dialogue. In Proceedings of the 2018 EMNLP Workshop SCAI: The 2nd International Workshop on Search-Oriented Conversational AI, pages 87-92, Brussels, Belgium. Association for Computational Linguistics.

Sam Wiseman, Stuart Shieber, and Alexander Rush. 2017. Challenges in data-to-document generation. In Proceedings of the 2017 Conference on Empirical Methods in Natural Language Processing, pages 2253-2263, Copenhagen, Denmark. Association for Computational Linguistics.

Sam Wiseman, Stuart Shieber, and Alexander Rush. 2018. Learning neural templates for text generation. In Proceedings of the 2018 Conference on Empirical Methods in Natural Language Processing, pages 3174-3187, Brussels, Belgium. Association for Computational Linguistics.

Thomas Wolf, Lysandre Debut, Victor Sanh, Julien Chaumond, Clement Delangue, Anthony Moi, Pierric Cistac, Tim Rault, R'emi Louf, Morgan Funtowicz, and Jamie Brew. 2019. Huggingface's transformers: State-of-the-art natural language processing. ArXiv, abs/1910.03771.

Yonghui Wu, Mike Schuster, Zhifeng Chen, Quoc V Le, Mohammad Norouzi, Wolfgang Macherey, Maxim Krikun, Yuan Cao, Qin Gao, Klaus Macherey, et al. 2016. Google's neural machine translation system: Bridging the gap between human and machine translation. arXiv preprint arXiv:1609.08144.

Yingce Xia, Fei Tian, Lijun Wu, Jianxin Lin, Tao Qin, Nenghai Yu, and Tie-Yan Liu. 2017. Deliberation networks: Sequence generation beyond one-pass decoding. In Advances in Neural Information Processing Systems, pages 1784-1794.

Jiacheng $\mathrm{Xu}$, Zhe Gan, Yu Cheng, and Jingjing Liu. 2020. Discourse-aware neural extractive text summarization. In Proceedings of the 58th Annual Meeting of the Association for Computational Linguistics, 
pages 5021-5031, Online. Association for Computational Linguistics.

Lili Yao, Nanyun Peng, Ralph Weischedel, Kevin Knight, Dongyan Zhao, and Rui Yan. 2019. Planand-write: Towards better automatic storytelling. In Proceedings of the AAAI Conference on Artificial Intelligence, volume 33, pages 7378-7385.

Tiancheng Zhao, Ran Zhao, and Maxine Eskenazi. 2017. Learning discourse-level diversity for neural dialog models using conditional variational autoencoders. In Proceedings of the 55th Annual Meeting of the Association for Computational Linguistics (Volume 1: Long Papers), pages 654-664, Vancouver, Canada. Association for Computational Linguistics.

\section{A Reproducibility}

Computing Infrastructure. Our model is built upon the PyTorch transformers-2.6.0 library by Wolf et al. (2019), with Pytorch-Lightning-0.7.3 (Falcon, 2019) for training routines. To improve training efficiency, we adopt mixed-precision floating point (FP16) computation using the 02 option of NVIDIA apex ${ }^{5}$. For both training and decoding, we utilize the Titan RTX GPU card with 24 GB memory.

Model Sizes. Our generation model has the same architecture as BART (Lewis et al., 2020) with $406 \mathrm{M}$ parameters. The content planner is built on top of $\mathrm{BERT}_{\text {base }}$, which has $110 \mathrm{M}$ parameters.

Running Time. Training the generation model takes 2.5 hours for argument, 5 hours for opinion, and 24 hours for news. The content planning model converges in 2.5-4 hours for three domains.

Decoding Settings. At inference time, we set $k=50$, temperature $=1.0$, and $p=0.9$ for nucleus sampling. The relatively large $k$ value is determined based on a pilot study, where we find that the refinement lacks diversity if $k$ is set to small values. Moreover, since the Transformer states need to be cached during autoregressive decoding and we perform three complete nucleus sampling runs in each refinement iteration, the GPU memory consumption is substantially increased. We therefore limit the maximum generation steps to 140 for argument, 243 and 335 for opinion and news.

Auto-Correction for Content Plan. When the content plan is predicted by the planner, the following post-processing steps are employed prior to the

\begin{tabular}{lllllll}
\hline & \multicolumn{2}{c}{ ARGGEN } & \multicolumn{2}{c}{ OPINION } & \multicolumn{2}{c}{ NEWS } \\
& sys & ref & sys & ref & sys & ref \\
\hline \# tokens & 133.3 & 130.2 & 228.5 & 246.3 & 424.5 & 435.5 \\
\# sentences & 8.6 & 5.6 & 11.1 & 8.2 & 19.2 & 13.5 \\
\# KP per sent. & 2.96 & 3.77 & 2.22 & 2.49 & 3.40 & 3.24 \\
KP distance & 2.61 & 2.95 & 5.70 & 6.02 & 3.76 & 5.08 \\
\hline
\end{tabular}

Table 6: Statistics on generated templates by our content planner. Tokens are measured in units of WordPiece (Sennrich et al., 2016). KP distance denotes the average number of tokens between two keyphrases that are in the same sentence. Both system output (sys) and human reference (ref) are reported.

masked template construction: (1) For a predicted keyphrase, its token positions are adjusted to a consecutive segment, so that the phrase is kept intact in the template. (2) If the predicted positions are not monotonic to the assignment ordering, they will be rearranged. For instance, if the assignment contains $\mathrm{KP}_{1} \triangleright \mathrm{KP}_{2}$, but position of $\mathrm{KP}_{2}$ is not strictly larger than that of $K P_{1}$, we instead place $\mathrm{KP}_{2}$ immediately after $\mathrm{KP}_{1}$ in the template. (3) Finally, since the planner and generator have different subword vocabularies, it is necessary to detokenize the predicted keyphrase assignment, and re-tokenize with the BPE vocabulary of the generator.

\section{B Template Construction Statistics}

We characterize the content planning results in $\mathrm{Ta}-$ ble 6. Specifically, we show the statistics on the automatically created templates based on the planner's output. As we can see, our system predicted templates approach human reference in terms of length, per sentence keyphrase count, and the average keyphrase spacing. Sentence segmentation occurs more often in our templates than the reference text, likely due to the frequent generation of [SEN] tokens.

\footnotetext{
${ }^{5}$ https://github.com/NVIDIA/apex
} 